J. RADIAT. RES., SUPPLEMEN'T, 339-346 (1991)

A Review of Forty-Five Years Study of Hiroshima and Nagasaki, Atomic Bomb Survivors

II. BIOLOGICAL EFFECTS

\title{
Interactive Effects between Radiation and Other Factors on Cancer Risk among A-Bomb Survivors - an Overview of RERF Studies
}

\author{
SUMINORI AKIBA \\ Department of Epidemiology, Radiation Effects Research Foundation, \\ Hiroshima 732, Japan
}

\begin{abstract}
Cancer/Epidemiology/Radiation/Smoking/Interaction
This paper reviews RERF cancer studies conducted to examine interactive effects between radiation and epidemiological risk factors, including smoking habits, occupation, dietary habits and $O B / G Y N$ history. Special emphasis is placed on a review of studies of interactive effects between cigarette smoking and radiation on lung cancer risk. Comments on these studies are made and proposals for future studies are presented.
\end{abstract}

\section{INTRODUCTION}

It is of great interest to examine whether radiation-induced cancer risk can be modified by other risk factors that are related to life-style, occupation, OB/GYN history and so on. However, it is not an easy task to pursue this question since it usually requires special efforts to collect information on these factors.

So far, RERF researchers and their collaborators have conducted many epidemiological cancer studies to investigate various factors other than radiation. More than half of them were conducted in the form of a case-control study nested in the cohort of A-bomb survivors. In a typical study, cancer cases and non-cancer controls are interviewed and information on a variety of factors, including smoking, drinking, occupation and OB/GYN history, is obtained. In some studies, concentrations of various serum components are compared between cases and controls. One of the major reasons for the frequent use of the case-control approach is to avoid the substantial difficulties involved in collecting information on numerous covariates from the entire cohort. The major purposes of those studies can be summarized as follows:

(i) to identify risk factors other than radiation,

(ii) to evaluate potential confounding biases in radiation-related cancer risk estimation introduced by these risk factors, and

(iii) to evaluate interactive effects between radiation and other risk factors.

This paper will review those studies to summarize our current knowledge on interactive effects between A-bomb radiation and other factors, including smoking habits, occupation, dietary habits and $\mathrm{OB} / \mathrm{GYN}$ history. 
Table 1-1. An example of multiplicative effect between two RRs

\begin{tabular}{ccc}
\hline Factor A & \multicolumn{2}{c}{ Factor B } \\
\hline & - & + \\
- & 1.0 & 4.0 \\
+ & 5.0 & 20.0 \\
\hline
\end{tabular}

Table 1-2. An example of additive effect between two cRRs

\begin{tabular}{ccc}
\hline Factor A & \multicolumn{2}{c}{ Factor B } \\
\hline & - & + \\
- & 0.0 & 3.0 \\
+ & 4.0 & 7.0 \\
\hline
\end{tabular}

\section{TERMINOLOGY}

There are several similar, and therefore confusing, terms related to interaction. To avoid misunderstanding, it seems appropriate to define the terminology at the outset.

The term "interaction" has, at least, three different meanings: (i) effect modification, (ii) synergism and (iii), in statistics, the necessity for a product term in a linear model ${ }^{1,2)}$. When a value of a risk indicator such as relative risk is dependent on the value of a factor other than the exposure of interest, the factor is called an effect modifier or, simply, a modifier. In other words, there is an effect modification caused by this factor.

In statistics, interaction is dependent on the model used in the analysis. There are two important models regarding interaction: a multiplicative and an additive one. An example of multiplicative model is shown in Table 1-1. In this example, the relative risk (RR) associated with exposure to factor $\mathrm{A}$ is 5 in both $\mathrm{B}+$ and $\mathrm{B}$ - groups (therefore, there is no modification of RR). Similarly, the RR associated with exposure to factor $B$ is 4 regardles of the exposure status to $\mathrm{A}$. And also, the RR that compares $\mathrm{A}+\mathrm{B}+$ group with $\mathrm{A}-\mathrm{B}-$ group is 20 . This $\mathrm{RR}$ value is exactly the same as the one obtained by multiplication of the two RRs, one associated with $\mathrm{A}$ and the other associated with $\mathrm{B}$. Thus, in this example, the two RRs act multiplicatively. However, if the two RRs do not behave multiplicatively, an interactive effect is considered to be at work. Table 1-2 gives an cxample of an additive model, where two risk indicators act 
additively. Here, risks are expressed using excess R R (eRR), which can be obtained by subtracting 1 from RR. In this example, the eRRs associated with factor $A$ and $B$ are 3 and 4, respectively, and the eRR that compares $\mathrm{A}+\mathrm{B}+$ with $\mathrm{A}-\mathrm{B}-$ is 7 , the sum of the two eRRs. In this model, an interactive effect is at work if the two eRRs do not act additively.

Synergism is the term used to describe a more-than additive effect resulting from joint exposure. For example, in a situation where an eRR related to joint exposure to A and $B$ is larger than the sum of two eRRs associated with $A$ and $B$, there is a synergism (and an interactive effect in an additive model). Therefore, if a multiplicative model fits data well, there is always a synergism.

\section{OVERVIEW OF CASE-CONTROL STUDIES CONDUCTED AT RERF}

At RERF, most of the studies which tried to examine interactions between radiation and epidemiological factors were conducted in the form of a case-control study. An exception was a series of studies on smoking and radiation, which will be reviewed in the following section. A list of the case-control studies conducted at RERF are given in Table 2. All of them were nested in LSS (consisting of 120,000 A-bomb survivors and unexposed subjects) or its clinical subsample, AHS population (consisting of 20,000 subjects). In more than half of these studies, the radiation doses or the distances from the hypocenter were matched between cases and controls. Such a procedure, taken to increase the statistical power in evaluation of interactive effects, made it impossible to estimate radiation-related cancer risks and to evaluate confounding biases possibly involved in the estimation of those risks. The fact suggests that the RERF researchers placed much more emphasis on evaluating interactive effects than on the assessment of confounding biases in estimation of radiation-related cancer risk. Such an emphasis seems justifiable since studies on interactive effects give us invaluable insights into the mechanisms of radiation carcinogenesis.

In the mid 1960s, Ishimaru conducted a case-control study of cancer of the cervix ${ }^{3)}$. Occupational history, OB/GYN history, life styles and socioeconomic factors were examined but none were found to be significantly related to cervical cancer risk. Apparently, no attempt was made in their study to evaluate the interaction between these factors and radiation.

As a part of the leukemia study series, Ishimaru et al. ${ }^{4)}$ conducted a case-control study of leukemia among the RERF cohort and obtained information on their occupational history by interviews. They found an approximately 2.5 fold increase of leukemia risk among those with history of probable occupational exposure to benzene or its derivatives and medical x-ray. The risk associated with atomic bomb exposure was not significant modified by those occupational exposures.

In the 1970s, Nakamura ${ }^{5)}$ conducted a case-control analysis of data on breast cancer for the period of 1958-69 and suggested the presence of a multiplicative effect between radiation and OB/GYN history. In the early 1980s another case-control study of breast cancer was conducted with the collaboration of U.S. NCI researchers ${ }^{6}$. In this study all the living cases diagnosed during the period of 1950-80 were interviewed. While the analysis of the entire data set obtained from the study is yet to be completed, a preliminary analysis suggested the presence of a 
multiplicative effect between radiation and $\mathrm{OB} / \mathrm{GYN}$ history. (C. Land, Personal communication)

In a recent study ${ }^{7}$, the levels of various hormones were determined for cancers of the breast, endometrium and ovary as well as their matched controls. This case-control study was nested in the AHS cohort. Estradiol, DHEAs, prolactin and other substances were measured using serum samples collected during the period of $1970-72$ and stored frozen since. Although analysis is yet to be completed, there seem to be complicated relationships between breast cancer risk and various hormones. Similar studies using stored serum samples are conducted for cancers of the thyroid and prostate ${ }^{7}$.

One of the most interesting topics in the field of cancer epidemiology is the relationship between nutrients and cancer risk. At RERF a few studies were conducted to examine the relationship. In a case-control study of colorectal cancer ${ }^{6}$, an interview survey was conducted to obtain dietary habits and other information to study the interaction between radiation and dietary habits. Unfortunately, the quality of data on dietary habits obtained from this study does not seem to be good enough to enable the evaluation of such an interaction. A similar case-control study of thyroid cancer is currently conducted ${ }^{\text {9) }}$. Recently, serum levels of nutrients in cases of cancer of the lung or stomach as well as their controls were determined ${ }^{10)}$ using stored serum samples collected in the periods of 1970-72 and 1977-79. In this study nested in the AHS population, selenium, zinc, beta-carotene, alpha-tocopherol and several other factors were measured. An interesting finding obtained from this study was an inverse relationship between body iron storage, which was determined by serum ferritin levels, and gastric cancer risk ${ }^{11)}$. The association was, however, concluded to be non-causal, being a consequence of chronic atrophic gastritis, which predispose to stomach cancer. No nutrients measured in this study was found to be a significant modifier of radiation-related cancer risk.

Probably, the single most important risk factor in cancer epidemiology is cigarette smoking. Therefore, it is not surprising that the interactive effect between smoking and radiation on lung cancer risk has been most extensively studied. Several groups of investigators addressed this issue using different approaches. A review of these papers will be given in the following section.

\section{INTERACTIVE EFFECTS BETWEEN SMOKING AND RADIATION ON LUNG CANCER RISK}

\section{A review of studies conducted at RERF}

There are five studies that evaluated the interactive effects between smoking and radiation on lung cancer risk (Table 2). In these studies, the cases were ascertained from various sources:

(i) autopsy records, which were used in the case-control study of Ishimaru et al. ${ }^{12)}$;

ii) the RERF mortality survey of atomic bomb survivors (LSS), which covers all the deaths across the country and which was utilized in the cohort studies of Prentice et al. ${ }^{13)}$ and of Shimizu et al. ${ }^{14)}$; and

iii) incidence surveys, based mainly on tumor and tissue registries in Hiroshima and Nagasaki areas, as used for the cohort study of Kopecky et al. ${ }^{15)}$ and in the case-control study of Blot et al. ${ }^{16,17)}$. 
Table 2. A list of cancer case-control studies at RERF

\begin{tabular}{|c|c|c|c|}
\hline Cancer & Investigators & $\begin{array}{l}\text { Research } \\
\text { Protocol }\end{array}$ & $\begin{array}{l}\text { Technical } \\
\text { Report }\end{array}$ \\
\hline leukemia* & Ishimaru et al. & - & TR 26-69 \\
\hline breast & Nakamura et al. & - & TR $\quad 9-77$ \\
\hline breast* & Land et al. & RP $14-79$ & - \\
\hline breast, endometrium, ovary* & Kabuto et al. & RP $11-85$ & - \\
\hline lung & Ishimaru et al. & - & TR $33-72$ \\
\hline lung & Akiba et al. & RP 15-81 & TR $7-85$ \\
\hline lung, stomach & Kabuto et al. & RP $10-85$ & TR $14-89$ \\
\hline colo-rectum* & Akiba et al. & RP $\quad 6.83$ & - \\
\hline uterine cervix & Ishimaru et al. & - & TR $16-65$ \\
\hline thyroid* & Yoshimoto et al. & RP $12-85$ & - \\
\hline thyroid* & Kabuto et al. & RP $11-85$ & - \\
\hline prostate* & Kabuto et al. & RP 11-85 & - \\
\hline
\end{tabular}

* Studies in which the radiation dose or the distance from hypocenter was matched.

Information on smoking habits was obtained mainly by next-of-kin interviews in two casecontrol studies. In cohort studies, data obtained from mail surveys and interviews conducted at the RERF clinics were consolidated and used.

On inspection, the crude data of these studies seem to support an additive RR model. For example, in the case-control study of Blot et al., the RR associated with smoking was 6.0 in men who smoked 11-20 cigarettes per day and the RR associated with exposure to a radiation dose (TR65DR) of 1.0 Gy or more was 3.5 while the RR associated with both exposures relative to non-smokers with a radiation dose of less than .1 Gy was 10.6. However, no study conducted so far has successfully rejected a multiplicative model. BEIR IV committee, which reviewed all of the RERF studies carefully and reanalyzed the data from one case-control study concluded that either a multiplicative or an additive RR model fits the data, with little preference ${ }^{18}$.

\section{Comments and proposal for future studies}

The RR model obtained from the analysis by BEIR IV committee predicts a smaller joint effect between radiation and smoking than expected from multiplicative RR models. In such a model, the RR associated with radiation is smaller in smokers than in non-smokers. Therefore, the observed sex difference in radiation-related lung cancer risk ( $R R$ was about two-fold higher in women than in men) can be explained by the interaction between smoking and radiation since more than $80 \%$ of Japanese men had once smoked cigarettes while less than $20 \%$ of Japanese women had $^{14-16)}$.

What should be noted in the data of A-bomb survivors is the relatively low RR of lung cancer associated with smoking, which is about 5 for male smokers. Relatively low RRs are observed not only in the RERF cohort but also in most of the Japanese studies ${ }^{19)}$, where the reported 
Table 3. RERF studies on the interactive effect between smoking and radiation on lung cancer risk

\begin{tabular}{|c|c|c|c|}
\hline \multirow{2}{*}{$\frac{\text { Investigators }}{\text { Ishimaru et al. }}$} & Study design & \multicolumn{2}{|c|}{$\begin{array}{c}\text { Source of cases } \\
\text { (observation period) }\end{array}$} \\
\hline & case-control study & autopsy records & $(1961-70)$ \\
\hline Prentice et al. & cohort study & mortality files & $(1963-78)$ \\
\hline Blot et al. & case-control study & incidence data & $(1971-80)$ \\
\hline Kopecky et al. & cohort study & incidence data & $(1963-80)$ \\
\hline Shimizu et al. & cohort study & mortality files & $(1963-85)$ \\
\hline
\end{tabular}

RRs are less than a half of those obtained from the studies conducted in the U.S. and western Europe $^{20)}$. The most significant among the several factors involved in the low RRs observed in Japan, is the cigarette shortage which lasted for about 5 years during and immediately after World War $\mathrm{II}^{21,22)}$. Thus, when this shortage was taken into account, the lung cancer risk in the Japanese was similar to that observed in the British physicians' cohort ${ }^{19)}$. As younger birth cohorts not affected by the shortage begin to reach the so-called cancer ages, the RRs associated with cigarette smoking becomes larger ${ }^{19)}$. In the analysis to be conducted in the future, attention should be given to this cigarette shortage problem.

Another problem involved in studies of cigarette smoking is the change in the proportion of smokers over time. In the mid 1960s, more than $80 \%$ of Japanese men were smokers and similar percentages were also observed among A-bomb survivors in Hiroshima and Nagasaki ${ }^{23)}$. Since then, the proportion of male smokers declined continuously and a recent survey recorded the proportion to be near $60 \%$. Considering this rapid decline in the proportion of male smokers, it seems necessary to step up an effort to monitor changes in smoking habits among A-bomb survivors. Although RERF has conducted mail surveys at least every ten years, that is not enough for the purpose of monitoring smoking habits. It seems necessary to devise another mechanism to monitor smoking habits.

There are several studies other than those conducted among A-bomb survivors where interactive effects between smoking and radiation on lung cancer were assessed. One of the most well-known studies, conducted among the cohort of Colorado Plateau uranium miners, reported multiplicative effects ${ }^{24,25)}$. Uranium miners are different from A-bomb survivors in several respects. For example, radiation exposure in the former is a continuous one to alpha particles while in the latter it was a one-time exposure to mainly low LET radiation ${ }^{16}$. In addition, in A-bomb survivors, most of the exposure to cigarette smoke started after exposure to radiation because of the cigarette shortage in the 1940s while the uranium miners were exposed to cigarette smoke and radon daughters concurrently. Whether the interaction of smoking and radiation differs between the two types of populations is yet to be determined. As a comparative study between the A-bomb population and the Colorado Plateau uranium miners, comparisons of histological distribution of lung cancer are currently conducted ${ }^{26)}$. 


\section{CONCLUDING REMARKS}

Epidemiological studies to examine interactive effects between radiation and other factors are important and should give us invaluable clues to understanding the mechanisms involved in radiation carcinogenesis. Studies at RERF conducted so far suggest that smoking and $O B / G Y N$ history are the most promising covariates for studies of such interactions. However, conventional epidemiological approaches have their own limitations in studying the mechanisms involved in carcinogenesis. On the other hand, remarkable progresses have recently been made in basic cancer research using techniques of molecular biology. For example, a loss of specific gene function was found to be important in the etiology of retinoblastoma and other cancers, including lung cancer $^{27,28)}$. Furthermore, a recent study revealed that heredity plays a more significant role in smoking-related lung cancer than it was previously thought to do. A study by Caporaso et al. ${ }^{29)}$ suggested that extensive metabolizers of debrisoquine were more likely to develop smoking related cancer lung cancer than poor metabolizers and the increased risk in the former was primarily for histological types strongly related to smoking. These recent developments in molecular biology and biochemical techniques seem to provide us with ample opportunities to advance our understanding of the mechanisms of radiation carcinogenesis. New epidemiological programs that facilitate the use of newly developed techniques in combination with epidemiological approaches are urgently needed.

\section{REFERENCES}

1. Last, J.M. (1988) A Dictionary of Epidemiology. Oxford University Press, Oxford.

2. Blot, W.J. and Day, N.E. (1979) Syenewrgism and interaction, Are they equivalent? Amer. J. Epidemiol. 110: $99-100$.

3. Ishimaru, T. (1965) Epidemiologic study of uterine cancer, Hiroshima. RERF TR 16-65.

4. Ishimaru, T., Okada, H., Tomiyasu, T., Tsuchimoto, T., Hoshino, T. and Ichimaru, M. (1971) Occupational factors in the epidemiology of leukemia in Hiroshima and Nagasaki. Amer. J. Epidemiol. 93(3): 21-29.

5. Nakamura, K., McGregor, D., Kato, H. and Wakabayashi, T. (1977) Epidemiologic study of breast cancer in A-bomb survivors. RERF TR 9-77.

6. Yamada, Y., Land, C.E. and Hayakawa, N. (1979) Interaction between radiation dose and host factors. An epidemiological case-control study of female breast cancer in atomic bomb survivors. RERF RP 14-79.

7. Kabuto, M., Neriishi, K., Soda, M., Akiba, S., Kato, H., Land, C.E., Morimoto, I., Izumi, M., Takemoto, T., Pike, M. (1985) Hormone status in relation to cancer: A prospective epidemiologic study using store sera. RERF RP 11-85.

8. Akiba, S., Land, C.E., Kinlen, L.J., Ershow, A.G., Nakatsuka, H. and Sekine, 1. (1983) A case-control study on colorectal cancer. RERF RP 6-83.

9. Ishimaru, T., Hoffman, D., Ershow, A.G., Land, C.E., Wakabayashi, T., Hayashi, Y. and Tsuda, N. (1985) Epidemiologic case-control study of thyroid cancer in Hiroshima and Nagasaki. RERF RP 12-85.

10. Kabuto, M., Neriishi, K., Shidoji, Y., Soda, M., Kato, H., Akiba, S., Blot, W.J., Prentice, R.L., Hosoya, N., Suzuki, T., Takemoto, T., Land, C.E. (1985) Nutrients in relation to cancer risk: A prospective epidemiologic study using store sera. RERF RP 10-85.

11. Akiba, S., Neriishi, K., Blot, W.J., Kabuto, M., Stevens, R.G., Kato, H. and Land, C.E. (1989) Serum ferritin and stomach cancer risk among A-bomb survivors. RERF TR 14-89.

12. Ishimaru, T., Chaik, R.W., Land, C.E., Steer, A. and Yamada, A. (1975) Lung cancer at autopsy in A-bomb 
survivors and controls, Hiroshima and Nagasaki, 1961-1970. II. Smoking, occupation, and A-bomb exposure. Cancer 36(5): 1723-1728.

13. Prentice, R., Yoshimoto, Y. and Mason, M. (1983) Relationship of cigarette smoking and radiation exposure to cancer mortality in Hiroshima and Nagasaki. J. Natl. Cancer Inst. 70: 611-622.

14. Shimizu, Y., Kato, H. and Schull, W.J. (1990) Studies of the mortality of A-bomb survivors: Mortality, 1950-1985, II: Cancer mortality based on the recent revised doses (DS86). Radiat. Res. 121: 120-141.

15. Kopecky, K., Yamamoto, T., Fujikura, T., Tokuoka, S., Monzen, T., Nishimori, I., Nakashima, E. and Kato, H. (1986) Lung cancer, radiation exposure and smoking among A-bomb survivors, Hiroshima and Nagasaki, 1950-80. RERF TR 13-86.

16. Blot, W.J., Akiba, S. and Kato, H. (1984) Ionizing radiation and lung cancer: A review including preliminary results from a case-control study among A-bomb survivors. In atomic bomb survivor Data: Utilization and analysis, R.L. Prentice and D.J. Thompson, eds. Philadelphia: Society for Industrial and Applied Mathematics.

17. Akiba, S., Yoshimoto, Y., Blot, W.J., Hayakawa, N. and Hoel, D.G. (1981) Case-control study of lung cancer among atomic bomb survivors. RERF RP 15-81.

18. Committee on the Biological Effects of Ionizing Radiation, National Research Council (1988) Health Effects of Radon and other internally deposited alpha-emitters (BEIR IV). Washington, DC: National Academy Press.

19. Mizuno, S., Akiba, S. and Hirayama, T. (1989) Lung cancer risk comparison among male smokers between the "Six-prefecture cohort"' in Japan and the British physicians' cohort. Jpn. J. Cancer Res. 80: 1165-1170.

20. A report of the Surgeon General (1982) The health consequence of smoking, "Cancer" U.S. Department of Health and Human Service. Office on Smoking and Health, Rockville, Maryland.

21. Shimizu, H. (1979) Smoking habits in Japan. Smoking and Health 1(3): 7. (in Japanese)

22. Tominaga, S. (1986) Smoking and cancer patterns and trends in Japan. In "Tobacco: A Major International Health Hazard," ed. D.G. Zaridze and R. Peto, IARC Scientific Publications No. 74, pp.103-113.

23. Kato, H., Johnson, K.G. and Yano, K. (1956) Mail survey of cardiovascular disease study, Hiroshima and Nagasaki. ABCC TR 19-66.

24. Archer, V.E., Wagoner, J.K. and Lundin, F.E. (1973) Uranium mining and cigarette smoking on man. J. Occup. Med. 15: 204-211.

25. Whittemore, A.S. and McMillan, A. (1983) Lung cancer mortality among U.S. Uranium miners: A reappraisal. J. Natl. Cancer Inst. 71: 489-499.

26. Fujikura, T., Tokunaga, M., Akiba, S., Shimosato, Y., Tokuoka, S., Tateishi, R., Nambu, S., Saccomano, G., Auerbach, O., Matthews, M., Greenberg, S.D., Carter, D., Land, C. and Mason, T. (1986) Pathology review of lung cancer among atomic bomb survivors and uranium miners. RERF RP 9-86.

27. Harbour, J.W., Lai, S.L., Whang-Peng, J., Gazdar, A.F., Minna, J.D. and Kaye, F.J. (1988) Abnormalities in structure and expression of the human retinoblastoma gene in SCLC. Science 241: 353-357.

28. Friend, S.H., Bernard, R., Rogelj, S., Weinberg, R.A., Rapaport, J.M., Albert, D.M. and Dryja, T.P. (1986) A human DNA segment with properties of the gene that predisposes to retinoblastoma and osteosarcoma. Nature 323: 643-646.

29. Caporaso, N.E., Tucker, M.A., Hoover, R.N., Hayes, R.B., Pickle, L.W., Issaq, H.J., Muschik, G.M., Green-Gallo, L., Buivys, D., Aisner, S., Resau, J.H., Trump, B.F., Tollerud, D., Weston, A. and Harris, C.C. (1990) Lung cancer and debrisoquine metabolic phenotype. J. Natl. Cancer Inst. 82: 1264-1271. 